\title{
Auch mit DOAK bleibt es kompliziert
}

\author{
Anhand zweier Fälle diskutierten in MMW 3/2017, S. 43-48, Vertreter von Neurologie, Kardiologie \\ und Geriatrie die Antikoagulation bei betagten Patienten. Erwartungsgemäß lagen die Empfeh- \\ lungen der Experten teils auseinander - und auch unsere Leser hatten noch Anmerkungen.
}

\begin{abstract}
VKA sind bei Niereninsuffizienz in der Regel kontraindiziert

- Mein Kommentar zu Fall 1: Eine chronische Niereninsuffizienz im Stadium II-III und eine offensichtlich bestehende Hepatopathie sind doch per se jeweils (!) eine klare Kontraindiktion für den Einsatz von Vitamin-K-Antagonisten (VKA). Der Einsatz von Diclofe-
\end{abstract}

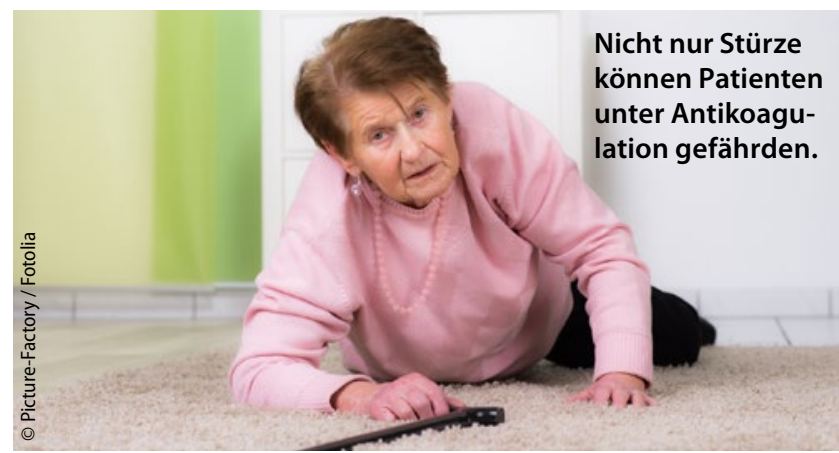

nac $2 \times 50 \mathrm{mg}$ und Pantoprazol $20 \mathrm{mg}$ imponiert reichlich verwegen. Eventuell ist auch überhaupt primär die beschriebene Anämie behandlungsbedürftig - in dem Fall am besten parenteral.

Auch beim Fall 2 ist eventuell die Anämie das primäre Problem. Ist eine orale Eisensubstitution hier überhaupt sinnvoll? Auch hier erscheint mir bei einer GFR von $56 \mathrm{ml} / \mathrm{min}$ der Einsatz von VKA eher heikel.

Wir alle haben in der Vergangenheit viel Unfug mit der VKA-Therapie getrieben. Das Problem ist die Tendenz zur Hyperkalzifikation, die man im Alltagsbetrieb ja nicht nur im Gefäßduplex, sondern auch im Echo besichtigen kann
- gerade bei den höheren Altersgruppen. Seit der Einführung direkter oraler Antikoagulanzien (DOAK) mit Zulassung bis zu GFR-Werten unter 30 ist es m.E. eher sinnlos, sich bei diesen Patienten noch mit VKA zu beschäftigen.

Thomas Engels, Facharzt für Innere

Medizin, D-78532 Tuttlingen

\section{Es fehlt der Spezialist für Vernunftmedizin}

_ Fall 1: Die Gabe von

Diclofenac bei Niereninsuffizienz ist unzulässig. Donepezil ist für die vaskuläre Demenz nicht geprüft und somit auch nicht indiziert. Diese Medikamente müssen abgesetzt werden

Das Sterberisiko eines 92-Jährigen lässt sich durch eine Antikoagulation nicht mehr nennenswert reduzieren. Bei gegebenen Risiken und lückenhafter Überwachung ist eine Indikation nicht vernünftig begründbar.

Fall 2: Eine Indikation für Pantoprazol und Mirtazapin lässt sich nicht sicher erkennen. Das Symptom Schwindel wird möglicherweise dadurch hervorgerufen. Bei fehlender Indikation gehört es abgesetzt. Die Antikoagulation mit Phenprocoumon ist unbefriedigend. Auch bei den DOAK ist nur bei konsequenter Einnahme ein Effekt zu erwarten. Aus Bequemlichkeitsgründen wären DOAK günstiger, eine bessere Wirkung und weniger Risiken sind nicht zu erwarten!
Fazit: Auch bei Hinzuziehen von drei Spezialisten bleiben wichtige Aspekte unberücksichtigt. Es fehlt der Spezialist für vernunftgesteuerte, praktikable Medizin.

Dr. Hans Georg Hoppenrath, Facharzt für Allgemeinmedizin, D-66484 Großsteinhausen

\section{Antwort der Autoren:}

Es wird deutlich, dass eine weitreichende Therapieentscheidung beim älteren Patienten gar nicht so einfach ist.

Der wichtigste Take-away der Diskussion scheint zu sein, dass bei einer chronischen Niereninsuffizienz im Stadium II (Kreatinin-Clearance 60-89 $\mathrm{ml} / \mathrm{min}$ ) bis Stadium III (30-59 $\mathrm{ml} / \mathrm{min}$ ) keine Kontraindikation für direkte orale Antikoagulanzien (DOAK) besteht. Die Dosis sollte allerdings entsprechend angepasst werden.

Das Sterberisiko eines 92-jährigen Patienten spielt tatsächlich eine untergeordnete Rolle in der Betrachtung der Indikation für eine orale Antikoagulation, allerdings ist auch klar, dass jede Ischämie eine Einschränkung der Funktionalität bedeutet und somit die Lebensqualität und auch die Selbstständigkeit der Patientin erheblich beinträchtigen kann. Vor diesem Hintergrund und auch aufgrund der vorliegenden Studiendaten ist eine Therapiebegrenzung rein aufgrund des Alters sicher nicht gerechtfertigt.

Prof. Dr. Markus Gosch, Nürnberg

Prof. Dr. Hans-Jürgen Heppner, Helios Klinikum Schwelm,

Dr.-Moeller-Str. 15, D-58332 Schwelm 